\title{
Observation of Magnetic Resonances in Electron Clouds in a Positron Storage Ring *
}

\author{
M. T. F. Pivi, * J. S. T. Ng, ${ }^{*}$ F. Cooper, D. Kharakh, F. King, \\ R. E. Kirby, B. Kuekan, C. M. Spencer, T. O. Raubenheimer, \\ and L. F. Wang \\ SLAC National Accelerator Laboratory, Menlo Park, CA 94025.
}

\begin{abstract}
The first experimental observation of magnetic resonances in electron clouds is reported. The resonance was observed as a modulation in cloud intensity for uncoated as well as TiN-coated aluminum surfaces in the positron storage ring of the PEP-II collider at SLAC. Electron clouds frequently arise in accelerators of positively charged particles, and severely impact the machines' performance. The TiN coating was found to be an effective remedy, reducing the cloud intensity by three orders of magnitude.
\end{abstract}

Key words: Electron cloud instability, secondary emission, positron storage rings, electron analyzer

PACS: 29.27.Bd, 29.20.db, 79.20.Hx, 52.20.Dq

\section{Introduction}

In the vacuum chamber of particle storage rings or accelerators, the formation of electron clouds may be initiated by photoelectrons released from surfaces and ionized residual gas molecules. The cloud density increases when electrons accelerated by the beam field impinge on the chamber wall and cause surface secondary emissions. Electron clouds, at sufficiently high density, can

\footnotetext{
ॠ Work supported by the US Department of Energy, Contract DE-AC0376SF00515.

* Corresponding authors: mpivi@slac.stanford.edu, jng@slac.stanford.edu.
} 
cause single- and coupled-bunch beam instabilities, emittance increase, pressure rise, and heat deposition at the wall, ultimately compromising a machine's performance. It is an important issue for many currently operating facilities with high-intensity positively charged particle beams, as well as in the design of the positron damping ring of the proposed International Linear Collider (ILC). Experimental and simulation results, as well as possible remedies, have been discussed and reviewed in a series of international workshops $[1,2]$.

The electron cloud effect is expected to be particularly severe in magnetic field regions. It has been studied in a dipole in the proton storage ring SPS [3], and in a wiggler in the KEK B-Factory [4]. We report detailed investigations of electron clouds and the observation of magnetic resonances in chicane dipole magnets in the positron storage ring of PEP-II. The experiment was designed to measure the total intensity, the horizontal distribution, and the longitudinal kinetic energy of the cloud electrons reaching the chamber wall for a variety of beam currents and magnetic field strengths, and to test possible mitigation methods.

\section{Experimental Setup}

The chicane was located in a dedicated $4.2 \mathrm{~m}$ long beamline in a PEP-II straight section. The magnets' $15 \mathrm{~cm}$ aperture accommodated both the beam pipe (10 cm outer diameter) and the detector assembly. The maximum field was $1.46 \mathrm{kG}$, matching the design strength of the ILC damping ring arc dipoles [5]. Each magnet was calibrated on a test bench to an accuracy of $0.03 \%$ in integrated field using a stretched-wire system. The magnet's power supply was stable at the $0.05 \%$ level over an 8 hour period [6]. The field-free sections were covered with current carrying windings producing a 20 Gauss solenoidal field to suppress electron cloud formation. The positron beam first passed through an uncoated aluminum chamber section along the center-line, encountering the first dipole after approximately $1.5 \mathrm{~m}$. When it reached the center of the second dipole, the trajectory had been deflected by approximately $3.5 \mathrm{~mm}$. Here, the inner surface of the aluminum chamber was coated with an $100 \mathrm{~nm}$ thin-film of TiN deposited by reactive sputtering from an axial $\mathrm{Ti}$ cathode in an $\mathrm{Ar} / 10 \% \mathrm{~N}_{2}$ atmosphere.

Each of the first three dipoles, separated center-to-center by $73 \mathrm{~cm}$, was instrumented with a retarding field analyzer (RFA) housed in an aluminum box welded on top of the beam pipe. Each RFA consisted of 3 layers of thin copper wire grids and one layer of stainless steel collectors positioned furthest from the beam line. The grids generated a highly uniform electric field that allowed measurement of the longitudinal kinetic energy (vertically along the field lines) of cloud electrons entering the detector region. The 17 strip col- 
lectors, $76.2 \mathrm{~mm}$ long and $2.54 \mathrm{~mm}$ wide, were placed on an horizontal plane length-wise along the beam direction with $0.51 \mathrm{~mm}$ gaps. An array of $2 \mathrm{~mm}$ diameter holes in the chamber wall, covering $15 \%$ of the local surface area, allowed shielding of the beam fields and detection of the electron cloud with minimal disturbance. Each collector was independently biased at $+45 \mathrm{~V}$. The detected signal current returned to ground via a load resistor. At the highest observed signal, this caused the bias voltage to "droop" by up to 1 volt; its effect on the RFA's collection efficiency was found to be negligible.

A photograph of the apparatus in the first chicane dipole is shown in Figure 1. The chamber wall exposed to direct synchrotron radiation beam was located on the $x>0$ side; $y$ is vertical. For the data presented here, PEP-II operated with 1722 bunches, with $6.65 \times 10^{10}$ positrons per bunch at an average beam current of $2500 \mathrm{~mA}$. The beam energy was $3.1 \mathrm{GeV}$. The beam bunches were $11.5 \mathrm{~mm}$ long (rms), with a spacing of $\tau_{b}=4.2 \mathrm{~ns}$. In the study of magnetic resonances in electron clouds in a dipole field, a useful quantity is the ratio of $\tau_{b}$ to the cyclotron period, $n=\tau_{b} / \tau_{c}$, where $\tau_{c}=2 \pi m_{e} \gamma / e B_{y}$, and $m_{e}$ is the electron's mass, $e$ its charge, $\gamma$ its Lorentz factor.

\section{$3 \quad$ Electron Cloud Build-up}

The number of electrons emitted from the surface is determined by the secondary electron yield (SEY). The SEY scales approximately as $1 / \cos (\theta)$, where $\theta$ is the incident angle with respect to the surface normal. For a fixed $\theta$, SEY increases rapidly as a function of incident energy until it reaches a maximum, and then decreases slowly at higher energies. The SEY parameters were measured in the laboratory using test samples, before and after exposure to positron beams in a setup installed at an upstream beamline location. The SEY maximum for uncoated aluminum surface was determined to be 3.2 at an incident energy of $300 \mathrm{eV}$, decreasing to 2.4 after beam exposure. While for a TiN-coated aluminum substrate, the maximum was 1.8 at $500 \mathrm{eV}$, reducing to 0.95 after beam exposure [7-9]. Both the laboratory measurement and the beam conditioning were done in a field-free environment.

During electron cloud build-up, low energy secondary electrons emitted from the surface were accelerated by the passing positron bunch. In the magnetic field-free case, the electrons would oscillate about the beam axis for 4 to 5 bunch crossings on average before impinging on the chamber wall. In the dipole field, the electrons were transversely localized, and they were constrained to move predominantly vertically along helical tracks. The cloud density stabilized within approximately 100 bunch crossings when the rate of electron production reached an equilibrium with the rate of loss due to re-absorption. The cloud electron flux at the chamber wall was measured by sampling the 
collector current at 1 second intervals, long after the build-up had reached equilibrium.

\section{Retarding Field Analyzer Measurements}

Because of the presence of the dipole field, the dynamics of the cloud electrons show significant spatial dependence with respect to the beam axis. The transversely segmented RFA was well-suited for this study. Some results are presented in this section.

\subsection{Secondary electron energy}

The longitudinal component of the secondary electron's energy, $K_{y}$, was measured by varying the retarding potential. The integrated $K_{y}$ spectra measured at selected RFA strips are shown in Figure 2 for uncoated aluminum surface at nominal beam current, $B_{y}=861 \mathrm{G}$. The selected strips extended over half of the RFA coverage to just beyond the beam axis, with strip-1 being the furthest from beam. The largest longitudinal kinetic energy gain occurred for electrons in the central region. For strip-10, $60 \%$ of the cloud electrons had longitudinal energy above $500 \mathrm{eV}$; while for strip-1, only $10 \%$ were above $50 \mathrm{eV}$.

The differential spectra shown in Figure 3 had been averaged over all strips. The observed peaks and other features are qualitatively consistent with expectations based on the chamber geometry and beam parameters [10]. For electrons detected beyond strip-6, the longitudinal energy distributions all peaked below $50 \mathrm{eV}$.

The secondary electron's energy gain was strongly position-dependent because the beam's electric field increased rapidly within the bunch's radius and decreased inversely with distance outside the bunch. The transverse position of the beam axis was inferred from the symmetric cloud density lateral distribution to lie between strips 9 and 10. Within the sensitivity of this measurement, there was no observable change in the $K_{y}$ distribution when the solenoid was powered off or on at $1 \mathrm{~A}$, or if the beam current changed by $10 \%$. Another set of measurement was taken at $B_{y}=904 \mathrm{G}$. The change was small but observable. At this dipole field, the average energy was higher: approximately $30 \%$ of the electrons had $K_{y}>300 \mathrm{eV}$ compared to $20 \%$. 


\subsection{Secondary electron lateral distribution}

Near the beam axis, a majority of the cloud electrons had $K_{y}>500 \mathrm{eV}$, which implies energies beyond the SEY peak. Electrons with large $K_{y}$ in general also had small $\theta$. The combined effect was a reduced secondary electron yield and a depleted electron cloud density at the center. A double-peak lateral distribution was therefore expected $[3,10]$. This was observed for aluminum surface as shown in the measured lateral distributions in Figure 4 at two dipole field values. It is especially clear for the $B_{y}=893 \mathrm{G}$ case, corresponding to $n=10.5$, a half-integer number of bunch-crossings per cyclotron period. The double-peak is less clear for the $n=10.0$ case, however. Indeed, for dipole fields greater than $425 \mathrm{G}(n=5)$ the following pattern was observed: there was clear depletion near the beam axis whenever the field values deviated from those corresponding to integer values of $n$. The double-peak was not observed at lower fields presumably because the electrons were less well-localized.

Measurements for TiN-coated surface are shown in Figure 5. Here a depletion near $x=0$ is discernable. But notice also the increase towards large positive- $x$ : this was the side of the chamber wall directly exposed to synchrotron radiation. Because of the large suppresion of secondary electron emission by the TiN coating, the signal detected in the RFA could have a significant contribution from photoelectrons emitted at the side-wall, making interpretation of the lateral distribution less straightforward.

\subsection{Dependence of electron cloud signal on beam current}

The electron cloud signals detected in selected collector strips are shown as a function of beam current in Figures 6 and 7. For uncoated aluminum surface, the cloud density growth in the center region $(-2 \mathrm{~mm}<x<2 \mathrm{~mm})$ stalled after an initial rise, and the ensuing increase was nearly absent. The large energy gain at this position, beyond the SEY peak, apparently caused a reduction in secondary electron production even at relatively low beam current. Further away from the center, at $x=29 \mathrm{~mm}$ for example, the energy gain was small and the build-up was almost linear with beam current. At $|x|=5 \mathrm{~mm}$, where the highest electron cloud signal was observed, the beam current dependence appeared to change at approximately $750 \mathrm{~mA}$ and $2200 \mathrm{~mA}$. Data for TiN-coated surface are qualitatively similar, although the strong suppression in the center region was not observed.

The observed beam current and lateral position variations indicate complex electron dynamics that depend on beam parameters and surface properties. This requires further study, and detailed simulations are being performed. 
Comparing the total signal for both surfaces, the TiN-coating had reduced the electron cloud intensity by at least 3 orders of magnitude at the nominal beam current of $2500 \mathrm{~mA}$.

\section{Magnetic Resonances in Electron Clouds}

Recent simulation studies revealed interesting cloud dynamics as the dipole field strength varied [11]. The phase of the electron's gyration motion with respect to the arrival time of the positron bunch varies with $B_{y}$ through the electron's cyclotron period. At resonance, the ratio $n=\tau_{b} / \tau_{c}$ takes on integer values, and the electron motion is in phase with the external force (momentum kick by the beam field). According to simulations using ILC parameters [11], the in-phase electrons, on average, gain more transverse momentum than the out-of-phase ones. And because most of the cloud electrons initially have energies below the SEY peak, the energy gain and the associated increase in $\theta$ result in an increase in secondary electron production. Thus, an enhancement in the electron cloud signal is expected at resonance.

We sought to observe this resonance effect at PEP-II by scanning $B_{y}$ in steps of 1 Gauss over a range of 0 to $1.1 \mathrm{kG}$. The measured electron cloud signals are shown as a function of the ratio $n$ in Figures 8 and 9 for uncoated and TiNcoated aluminum surfaces, respectively. For uncoated aluminum surface, data from the collector strip furthest away from the beam $(x=29 \mathrm{~mm})$ showed clear resonance peaks at the expected integer $n$ values. At collector strips closer to the beam axis $(x=0)$, the peaks showed a double-spike structure. This effect was so severe that at $x= \pm 5 \mathrm{~mm}$, the signal enhancement had shifted to half-integer values of $n$. This was not observed for TiN-coated surface, where resonances occurred for integer $n$ (at large $n$ ) for all collector strips.

The double-spike feature was observed in simulations for uncoated aluminum surfaces when space charge forces become important [11]. From Figures 6 and 8, it can be seen that the effect was most significant at $|x|=5 \mathrm{~mm}$, where the observed electron cloud signal, and thus the inferred cloud density, was the highest. On the other hand, single resonance peaks were preserved where the observed cloud signal was small, for TiN-coated aluminum surface, and for uncoated aluminum at transverse locations away from the center (at $x=29 \mathrm{~mm}$ for example.) This qualitative agreement shows that the ongoing detailed simulation study is expected to yield insights into the complex dynamics caused by a strong space charge force in highly non-uniform electron clouds.

Also shown in the two figures are comparisons of collector signals at equal but opposite transverse distances from the beam axis. For uncoated aluminum, the 
cloud signals appeared symmetric. For TiN-coated surface, the signal on the side exposed to the direct synchrotron radiation typically showed an enhancement of $10 \%$ to $20 \%$. The signal was weak and it was susceptible to systematic effects, especially at very small and very large dipole fields. For clarity, only data within the $2.5<n<11.5$ range are shown in Figure 9 .

\section{Discussion}

The measurements represent a detailed data set on the complex dynamics of the electron cloud and its interaction with a dipole field. To gain further insight, a validated simulation is needed. Thus far our simulation efforts have met with limited success. In particular, it is difficult to simulate the observed magnetic resonances phenomenon in the central region, although the measurements further away from the beam axis could be modeled reasonably well. Detailed and time-consuming three-dimensional models including strong nonuniform space charge forces are perhaps required.

An important component in the simulation is the SEY which must be supplied by measurements. However, all measurements done so far had been carried out in field-free environments. A dipole field could conceivably affect the SEY. For example, the probabilities of gyrating electrons causing secondary emissions could be different from those electrons impinging directly on the surface due to the difference in the distribution of incident angles and the increased path length within the surface material. There are now attempts to measure the SEY in a strong dipole field. These efforts should improve the accuracies and reliabilities of future simulations.

\section{Conclusion}

For future work, the long term stability of the TiN coating will be studied. Complementary mitigation techniques will also be tested. Two more beam chambers, one with a triangular groove profile on the inner surface to trap low energy electrons, and one with TiZrV Non-Evaporable Getter (NEG) coating which has a lower initial maximum SEY, have been designed. The grooved chamber has been fabricated and it is being tested using the apparatus described here at the new CesrTA experimental facility [12].

In summary, electron cloud dynamics in a dipole magnetic field were investigated in detail using a transversely segmented RFA. TiN coating was found to reduce the cloud density by more than three orders of magnitude. Magnetic 
resonances were observed. These results could be exploited to mitigate the impact of electron clouds in future colliders.

\section{Acknowledgments}

We thank C. Celata and M. Furman of LBNL for pointing out the possible existence of magnetic resonances. We also thank the support staff at SLAC for their contributions to the design, construction, and calibration of the apparatus. A. Fisher and S. Anderson made careful magnetic measurements. This work would not have been possible without the dedicated efforts of D. Arnett, P. Bellomo, M. Hayes and his staff, A. Kacharovsky, J. J. Lipari, J. Olszewski, B. Smith, and the PEP-II operations team. We also thank F. J. Decker, S. DeBarger, J. Seeman, M. Sullivan, U. Wienands, and W. Wittmer for their support during commissioning of the experiment. Work supported by the U.S. Department of Energy under contract number DE-AC02-76SF00515.

\section{References}

[1] Various review articles, in: H. Fukuma et al. (Ed.), Proc. Int. Workshop on Electron-Cloud Effects, Daegu, Korea, 2007, and references therein.

[2] Section 2, in: K. Ohmi, M. Furman (Eds.), ICFA Beam Dynamics Newsletter No.33, 2004.

[3] J. M. Jimenez et al., LHC Project Report 632 (2003).

[4] H. Fukuma, in: Proc. 31st Advanced ICFA Beam Dynamics Workshop on Electron-Cloud Effects, Napa, California, 2004.

[5] Chapter 2.4, in: N. Phinney et al. (Ed.), International Linear Collider Reference Design Report, Vol. 3, 2007, SLAC-R-857.

[6] The magnets were powered by Lambda EMI Model ESS supplies. An apparent calibration shift reported in our preliminary analysis, see for example, Pivi et al., Proc. $11^{\text {th }}$ European Part. Acc. Conf., 2008, MOPP63, was due to a software error and has since been corrected.

[7] M. Pivi et al., in: Proc. $11^{\text {th }}$ European Part. Acc. Conf., Genoa, Italy, 2008, MOPP064; also see SLAC-PUB-14000.

[8] F. Le Pimpec et al., J. Vac. Sci. Tech. A23(6) (2005) 1610.

[9] F. Le Pimpec et al., Nucl. Instr. Meth. A 551 (2005) 187.

[10] L. F. Wang, Phys. Rev. ST Accel. Beams 5 (2002) 124402. 
[11] C. Celata et al., Phys. Rev. ST Accel. Beams 11 (2008) 091002.

[12] M. A. Palmer et al., in: Proc. Part. Acc. Conf., Vancouver, Canada, 2009, FR1RAI02. 

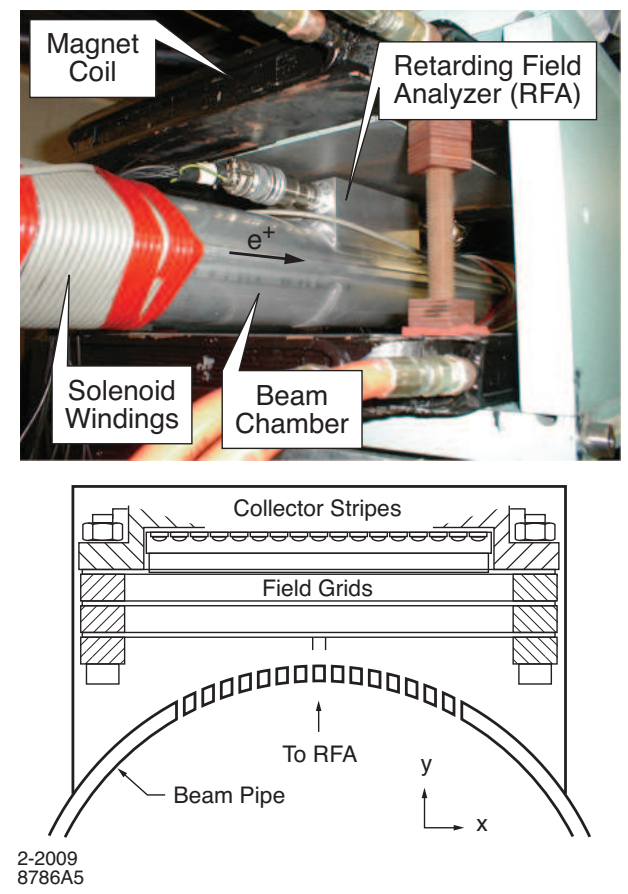

Fig. 1. Photograph of the apparatus in the first chicane dipole of the electron cloud experiment at PEP-II. A cross section schematic of the electron detector is also shown.

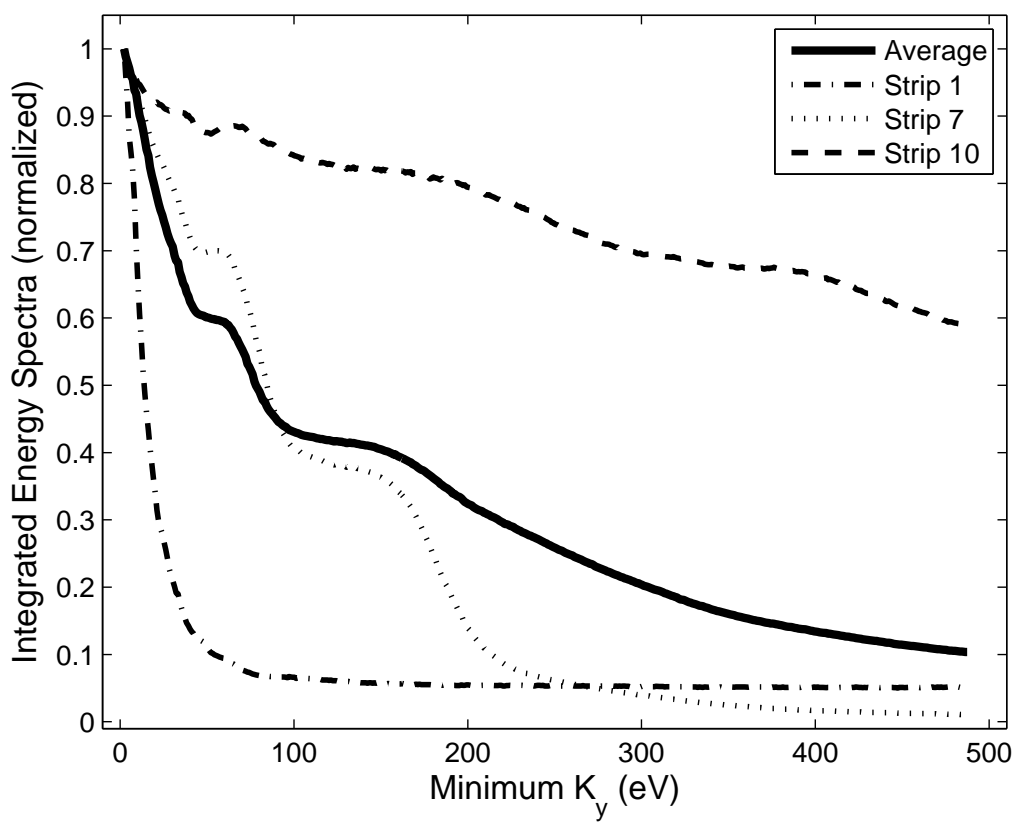

Fig. 2. Integrated energy spectra as a function of retarding potential energy for selected RFA strips and the average over all strips, for uncoated aluminum surface at $B_{y}=861 \mathrm{G}$. The beam axis lies between strips 9 and 10 . 


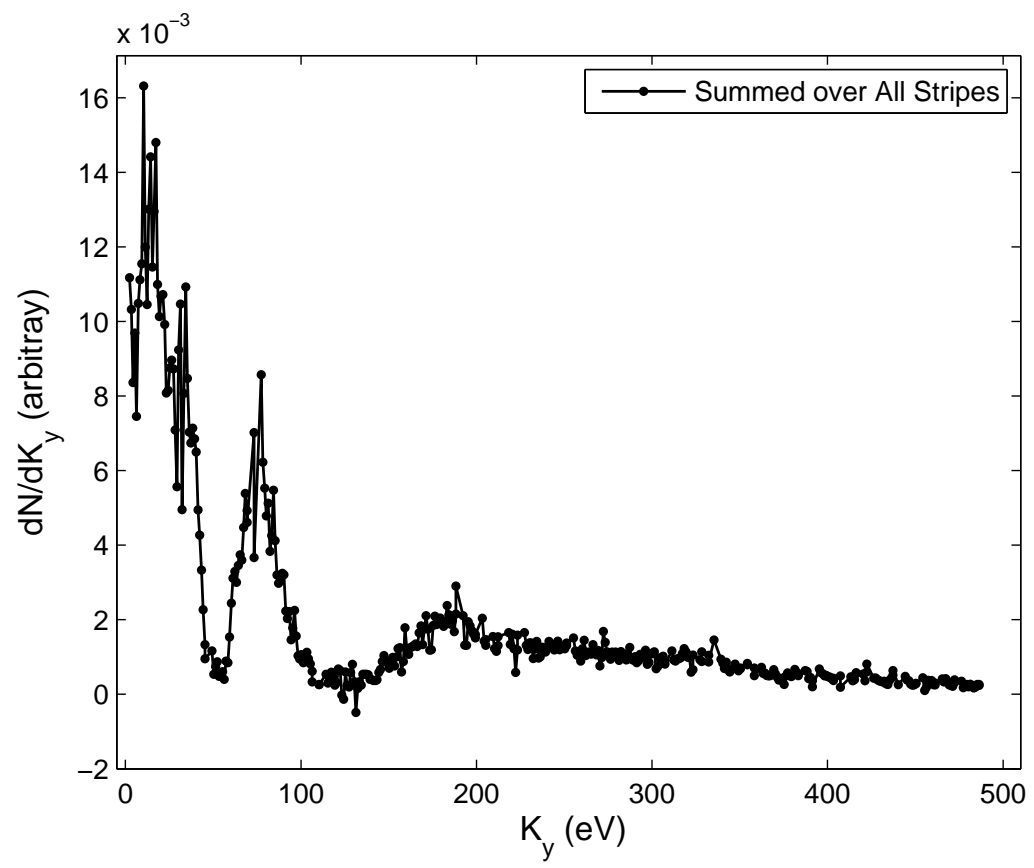

Fig. 3. Differential energy spectrum using data averaged over all RFA strips for uncoated aluminum surface; $B_{y}=861 \mathrm{G}$.

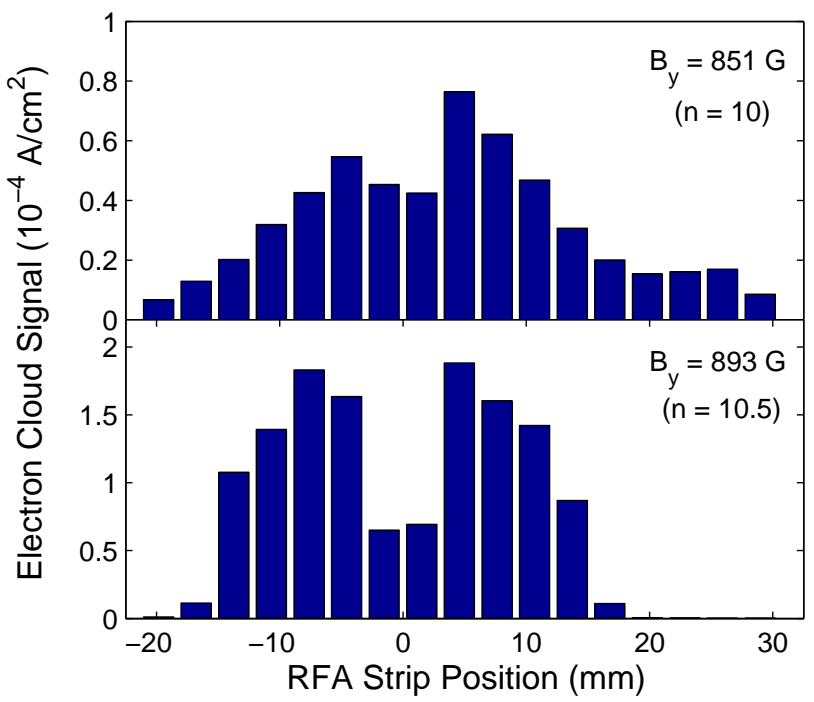

Fig. 4. Lateral distribution of electron cloud signal for aluminum surface at selected dipole fields. 


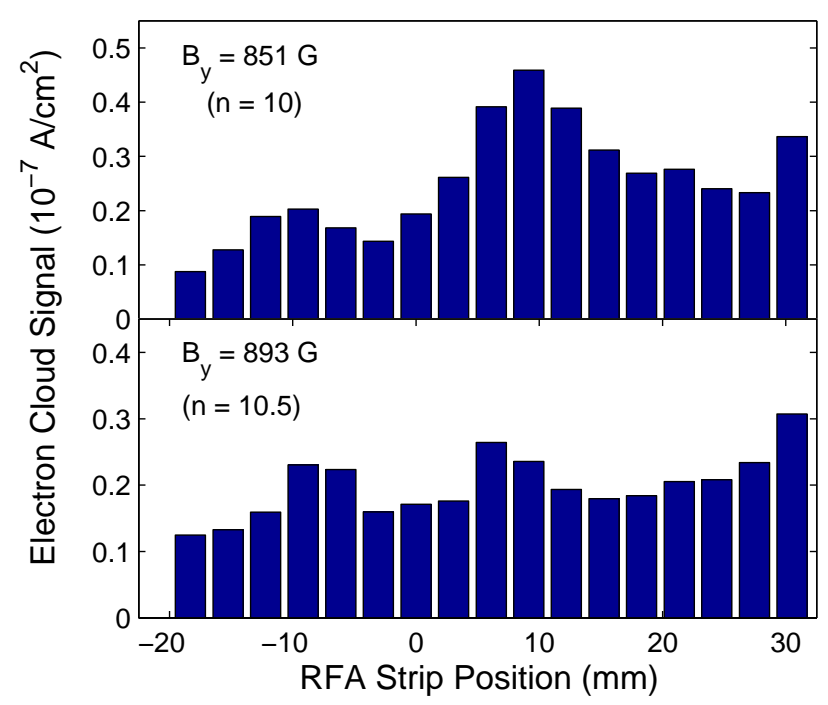

Fig. 5. Lateral distribution of electron cloud signal for TiN-coated surface at selected dipole fields.

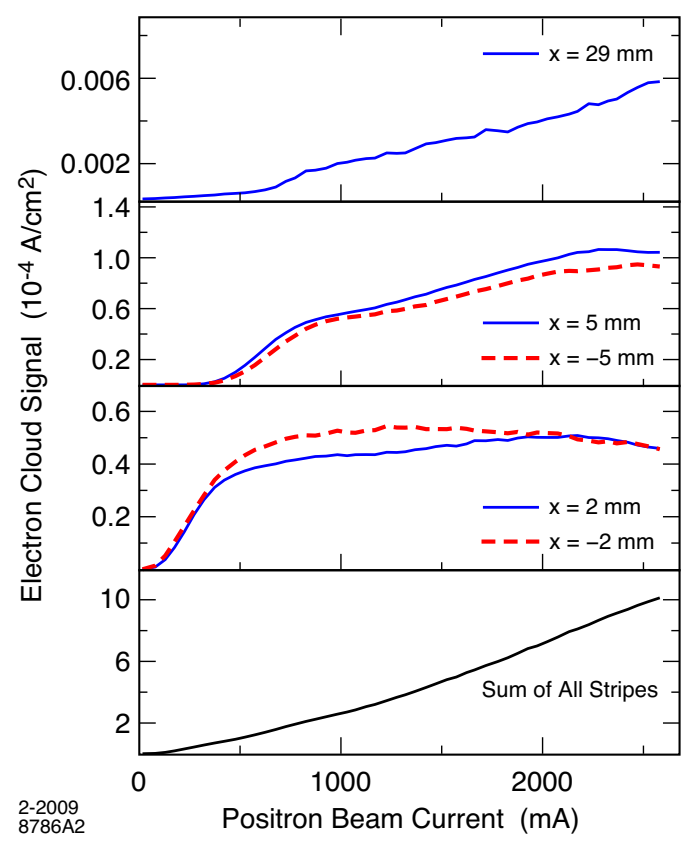

Fig. 6. Electron cloud signal as a function of beam current for uncoated aluminum surface at $B_{y}=861 \mathrm{G}$. Data from 5 selected RFA collector strips as well as the total signal summed over all 17 collectors are shown. 


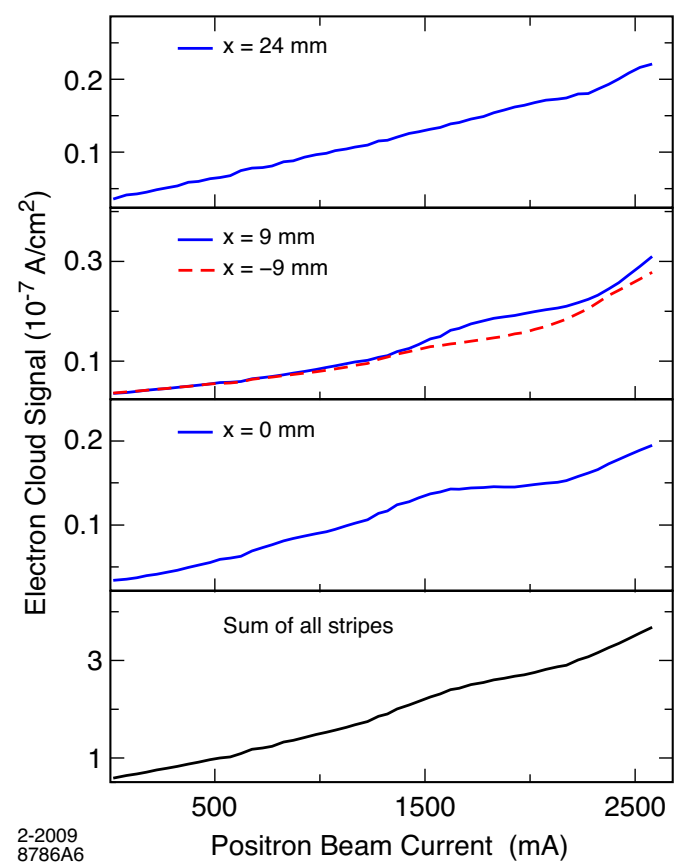

Fig. 7. Same as Figure 6 for TiN-coated surface for 4 selected collector strips, and note the factor of 1000 change in scale. 


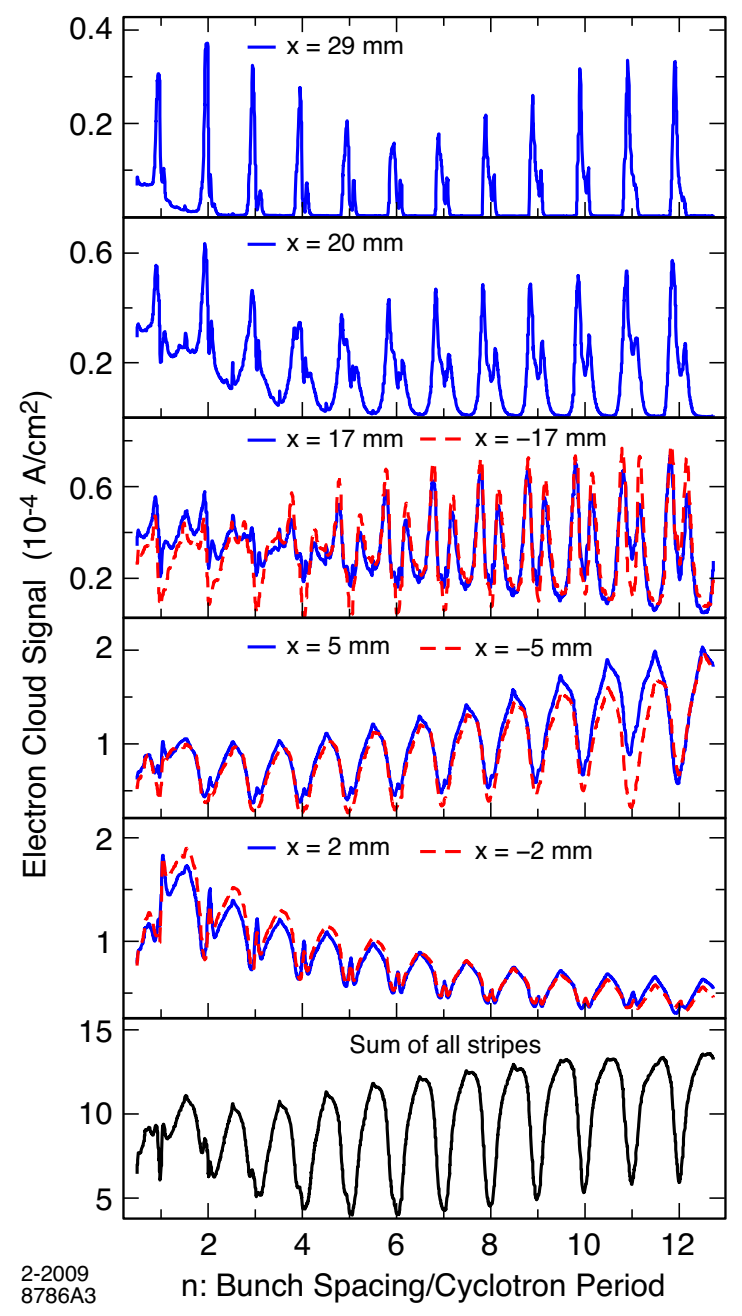

Fig. 8. Electron cloud signal from uncoated aluminum surface as a function of the ratio $n$ at $2500 \mathrm{~mA}$ beam current. Data from 8 selected collector strips as well as the total signal summed over all 17 collectors are shown 


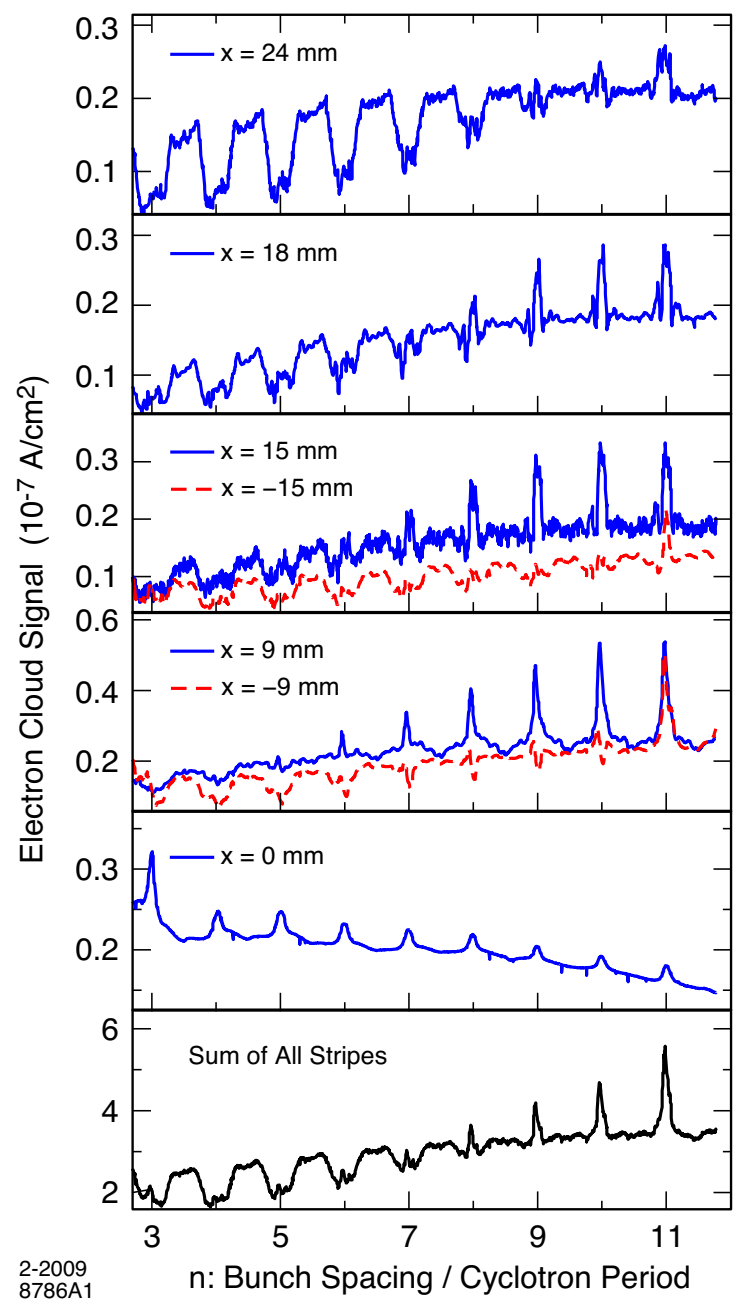

Fig. 9. Same as Figure 8 for TiN-coated surface for 7 selected collector strips, and note the factor of 1000 change in scale. 